\title{
HDACs confer glucagon responsiveness to FoxO for gluconeogenesis in the liver
}

\author{
Michihiro Matsumoto
}

Received: 22 August 2011/Published online: 21 September 2011

(C) The Japan Diabetes Society 2011

In mammals deprived of food, induction of hepatic glucose production (HGP) is important to ensure energy homeostasis, including prevention of hypoglycemia in response to the energy demands [1], and is especially critical in sites that predominantly utilize glucose as an energy source, such as the brain, red blood cells, and renal medulla. Glucose homeostasis is maintained through tight regulation of glucose production (i.e., glycogenolysis and gluconeogenesis) in the liver during the state of fasting and uptake of glucose in peripheral tissues under the fed condition. The pancreatic hormones glucagon and insulin are central to this regulation. During fasting, glucagon enhances HGP to maintain euglycemia. Conversely, when nutrients are increased after a meal, insulin blunts HGP and increases glucose uptake in the skeletal muscle and adipose tissue to prevent hyperglycemia. However, these processes are dysregulated in type 2 diabetes, resulting in the development of hyperglycemia [2]. The aberrant increase of HGP during the postabsorptive state in diabetic patients is primarily due to suppression failure by insulin, resulting primarily from excessive gluconeogenesis rather than glycogenolysis [3].

Gluconeogenesis is mainly regulated at the transcriptional level of rate-limiting gluconeogenic enzymes, such as glucose-6-phosphatase (G6pc; G6Pase) and phosphoenolpyruvate carboxykinase (Pck1; PEPCK) through hormone-dependent modulation of transcriptional regulators, including the transcription factors for FoxO (forkhead box O), CREB (cAMP response element binding protein),

\footnotetext{
M. Matsumoto ( $\square)$

Department of Molecular Metabolic Regulation,

Diabetes Research Center, National Center for Global Health

and Medicine, 1-21-1 Toyama, Shinjuku-ku,

Tokyo 162-8655, Japan

e-mail: mmatsumoto@ri.ncgm.go.jp
}

HNF $4 \alpha$ hepatocytes nuclear factor $4 \alpha$ and glucocorticoid receptor, and transcriptional coactivators, such as PGC- $1 \alpha$ (peroxisome proliferator-activated receptor gamma coactivator-1 alpha), CRTC2 (CREB-regulated transcription coactivator 2), and the histone acetyltransferase CBP (CREB-binding protein) [4].

Glucagon stimulates the induction of gluconeogenic genes through the following signaling pathway: cAMP, a second messenger of the glucagon receptor, leads cAMPdependent protein kinase (PKA)-mediated activation of the CRTC2/CREB complex to induce PGC- $1 \alpha$, which in turn coactivates the FoxO and HNF4 $\alpha$ transcription factors on the G6pc and Pck1 promoters (Fig. 1).

Insulin blunts gluconeogenic gene induction by affecting the same pathway. This is mediated mainly through the serine/threonine kinase Akt, which inactivates FoxO and PGC- $1 \alpha$ via direct phosphorylation, as well as CRTC2 via SIK (salt-inducible kinase) 1/2 [5] (Fig. 1).

The FoxO family of transcription factors, mainly FoxO1 and FoxO3 in mammalian liver [6], plays pivotal roles in regulating gluconeogenesis, as shown by studies on in vivo loss of and gain of function study; FoxO1 loss of function in the liver reduces HGP by inhibiting gluconeogenesis and glycogenolysis, resulting in neonatal and fasting-induced hypoglycemia [6], whereas its gain of function impairs hormonal regulation of HGP, including gluconeogenesis [7]. FoxO is regulated by insulin; Akt-dependent phosphorylation promotes its nuclear exclusion and inactivates FoxO1, leading to the reduction of gluconeogenic genes [8]. FoxO is also controlled by cAMP, which promotes its dephosphorylation and nuclear retention in cultured hepatocytes [8]. In vivo, FoxO1 loss of function in the liver attenuates gluconeogenic gene induction and HGP during fasting [6]. Although these findings indicate that FoxO is indispensable for cAMP-dependent induction of gluconeogenic genes, the 


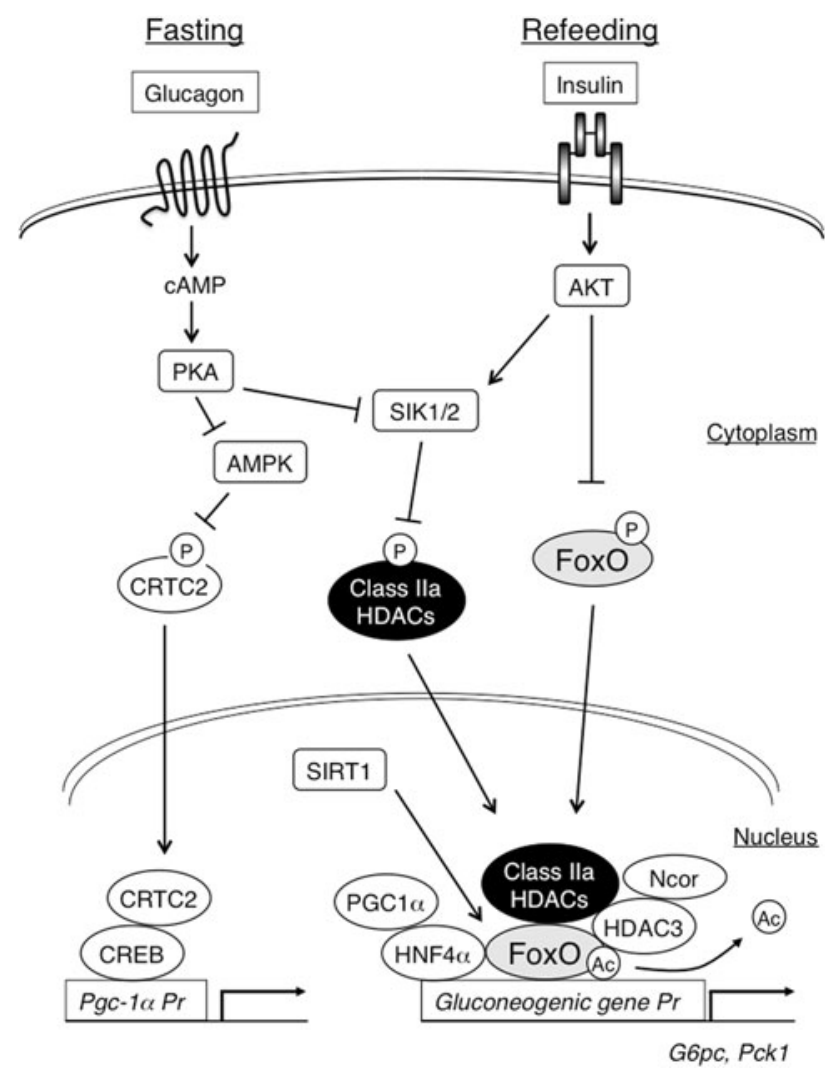

Fig. 1 Model for glucagon-dependent activation of FoxO by class IIa HDACs in mouse hepatocytes. During fasting, glucagon promotes dephosphorylation and nuclear translocation of class IIa HDACs through PKA-dependent inhibition of AMP-dependent protein kinase family members $(\mathrm{SIK} 1 / 2)$ in hepatocytes. In the nucleus, they function as scaffolds and form a transcriptional complex that includes HDAC3, Ncor, and FoxO on gluconeogenic gene promoters, resulting in HDAC3-mediated deacetylation and activation of FoxO. After a meal, insulin-induced activation of SIK1/2 promotes phosphorylation and nuclear exclusion of class IIa HDACs, leading to termination of a gluconeogenic program in cooperation with Akt-mediated inactivation of FoxO

mechanism by which cAMP activates FoxO to switch on its gluconeogenic program remains to be elucidated. The $\mathrm{NAD}^{+}$(nicotinamide adenine dinucleotide)-dependent protein deacetylase SIRT1, a member of class III histone deacetylases (HDACs), can activate FoxO by direct deacetylation [9]. However, there is no evidence suggesting cAMP-dependent activation of SIRT1 to date.

In Cell, Mihaylova et al. [10] clarify the mechanism of FoxO-mediated gluconeogenesis induced by glucagon/ cAMP during fasting. They identify class IIa HDACs as the key mediators. Another group has simultaneously reported that a similar signaling pathway is conserved in the fruit fly Drosophila melanogaster [11]. Mihaylova et al. found that by using genetic and biochemical loss-of-function approaches, class IIa HDAC4/5/7 are phosphorylated and excluded from the nucleus by AMPK family kinases, such as AMPK and SIK1/2, by treatment of the antidiabetic drug metformin or insulin in hepatocytes. In response to fasting or glucagon treatment, class IIa HDACs are dephosphorylated and translocated into the nucleus, where they serve as scaffolds for HDAC3, a catalytically active class I HDAC. In turn, HDAC3 deacetylates and activates FoxO, leading to gluconeogenic gene induction (Fig. 1). These findings unveil the mechanism by which glucagon/cAMP acutely activates FoxO, providing an advanced molecular basis for how FoxO exerts the counter-regulatory effects of glucagon and insulin on hepatic gluconeogenesis [6].

Class IIa HDACs are critical components not only to conferring glucagon responsiveness to FoxO for gluconeogenesis, but also in maintaining whole-body glucose homeostasis. The critical contribution of class IIa HDACs to hyperglycemia in the insulin-resistant state of the rodent model of type 2 diabetes is evinced by the phenotype of shRNA-mediated depletion of class IIa HDACs in the liver, hyperacetylation of FoxO, decreased expression of gluconeogenic FoxO target genes, and amelioration of hyperglycemia. These results apparently indicate that class I/IIa HDAC inhibitors may be useful as therapeutics for treating diabetes. As HDAC inhibitors are currently in development as potential anti-cancer drugs by pharmaceutical companies worldwide [12], their potential as therapeutics to treat diabetes should be investigated in the near future.

The Mihaylova study raises a number of important issues that should be addressed to understand the FoxOmediated metabolic regulation. Although acetylation as well as phosphorylation is critical to regulating the subcellular localization and activity of FoxO, how these modifications change and interplay under physiological or disease conditions in vivo remains unclear. The relative contribution of class IIa HDACs and SIRT1 to deaceylation-mediated FoxO activation, especially in insulin resistance, should be clarified from the therapeutic aspect of type 2 diabetes. It will also be important to know whether class IIa HDAC-mediated FoxO regulation is restricted to the liver. If it is not, it might be critical for FoxO biology and drug intervention to identify how this regulatory mechanism affects the ability of FoxO to control pleiotropic biological functions (i.e. proliferation, differentiation, anti-oxidative stress, longevity, as well as metabolism) in various tissues [13].

\section{References}

1. Cahill GF Jr. Fuel metabolism in starvation. Annu Rev Nutr. 2006;26:1-22.

2. Biddinger SB, Kahn CR. From mice to men: insights into the insulin resistance syndromes. Annu Rev Physiol. 2006;68:123-58. 
3. Rizza RA. Pathogenesis of fasting and postprandial hyperglycemia in type 2 diabetes: implications for therapy. Diabetes. 2010;59(11):2697-707.

4. Altarejos JY, Montminy M. CREB and the CRTC co-activators: sensors for hormonal and metabolic signals. Nat Rev Mol Cell Biol. 2011;12(3):141-51.

5. Koo SH, et al. The CREB coactivator TORC2 is a key regulator of fasting glucose metabolism. Nature. 2005;437(7062):1109-11.

6. Matsumoto $M$, et al. Impaired regulation of hepatic glucose production in mice lacking the forkhead transcription factor Foxo1 in liver. Cell Metab. 2007;6(3):208-16.

7. Nakae J, et al. Regulation of insulin action and pancreatic betacell function by mutated alleles of the gene encoding forkhead transcription factor Foxo1. Nat Genet. 2002;32(2):245-53.
8. Nakae J, et al. The forkhead transcription factor Foxo1 (Fkhr) confers insulin sensitivity onto glucose-6-phosphatase expression. J Clin Invest. 2001;108(9):1359-67.

9. Schwer B, Verdin E. Conserved metabolic regulatory functions of sirtuins. Cell Metab. 2008;7(2):104-12.

10. Mihaylova MM, et al. Class IIa histone deacetylases are hormone-activated regulators of FOXO and mammalian glucose homeostasis. Cell. 2011;145(4):607-21.

11. Wang B, et al. A hormone-dependent module regulating energy balance. Cell. 2011;145(4):596-606.

12. Lane AA, Chabner BA. Histone deacetylase inhibitors in cancer therapy. J Clin Oncol. 2009;27(32):5459-68.

13. Accili D, Arden KC. FoxOs at the crossroads of cellular metabolism, differentiation, and transformation. Cell. 2004;117(4):421-6. 